\title{
Gamma-glutamyltransferase activity in exosomes as a potential marker for prostate cancer
}

\author{
Kyojiro Kawakami ${ }^{1}$, Yasunori Fujita ${ }^{1}$, Yoko Matsuda ${ }^{2}$, Tomio Arai ${ }^{2}$, Kengo Horie ${ }^{3}$, Koji Kameyama ${ }^{3}$, Taku Kato ${ }^{3}$,
} Koichi Masunaga ${ }^{4}$, Yutaka Kasuya ${ }^{4}$, Masashi Tanaka ${ }^{5}$, Kosuke Mizutani $^{3^{*}}$, Takashi Deguchi ${ }^{3}$ and Masafumi Ito ${ }^{1^{*}}$

\begin{abstract}
Background: Exosomes or extracellular vesicles have the potential as a diagnostic marker for various diseases including cancer. In order to identify novel exosomal markers for prostate cancer (PC), we performed proteomic analysis of exosomes isolated from PC cell lines and examined the usefulness of the marker in patients.

Methods: Exosomes isolated by differential centrifugation from the culture medium of androgen-dependent LNCaP prostate cancer cell line and its sublines of partially androgen-independent C4, androgen-independent C4-2 and bone metastatic C4-2B were subjected to iTRAQ-based proteomic analysis. Exosomes were also isolated by immunocapture and separated by size exclusion chromatography and density gradient centrifugation. Protein expression was determined by Western blot analysis. GGT activity was measured using a fluorescent probe, $y$-glutamyl hydroxymethyl rhodamine green (gGlu-HMRG). Immunohistochemical analysis of tissues was performed using anti-GGT1 antibody.

Results: Among proteins upregulated in C4-2 and C4-2B cells than in LNCaP cells, we focused on gammaglutamyltransferase 1 (GGT1), a cell-surface enzyme that regulates the catabolism of extracellular glutathione. The levels of both GGT1 large and small subunits were elevated in exosomes isolated from C4-2 and C4-2B cells by differential centrifugation and by immunocapture with anti-CD9 or -prostate-specific membrane antigen (PSMA) antibody. In cell lysates and exosomes, GGT1 expression correlated with GGT activity. Size exclusion chromatography of human serum demonstrated the presence of GGT activity and GGT1 subunits in fractions positive for CD9. Density gradient centrifugation revealed the co-presence of GGT1 subunits with CD9 in exosomes isolated by differential centrifugation from human serum. Since GGT activity correlated with GGT1 expression in serum exosomes isolated by differential centrifugation, we measured serum exosomal GGT activity in patients. Unexpectedly, we found that serum exosomal GGT activity was significantly higher in PC patients than in benign prostatic hyperplasia (BPH) patients. In support of this finding, immunohistochemical analysis showed increased GGT1 expression in PC tissues compared with BPH tissues.
\end{abstract}

Conclusions: Our results suggest that serum exosomal GGT activity could be a useful biomarker for PC.

Keywords: Exosome, ү-glutamyltransferase 1, ү-glutamyl transpeptidase, Prostate cancer, Benign prostatic hyperplasia, Diagnostic marker

\footnotetext{
*Correspondence: mizutech@gifu-u.ac.jp; mito@tmig.or.jp

${ }^{3}$ Department of Urology, Gifu University Graduate School of Medicine, 1-1

Yanagido, Gifu, Gifu 501-1193, Japan

${ }^{1}$ Research Team for Mechanism of Aging, Tokyo Metropolitan Institute of

Gerontology, 35-2 Sakae-cho, Itabashi-ku, Tokyo 173-0015, Japan

Full list of author information is available at the end of the article
} 


\section{Background}

Exosomes or extracellular vesicles (EV) are microvesicles with a diameter of 40-150 $\mathrm{nm}$ that are secreted from various cells [1]. Numerous proteins, miRNAs, RNAs and DNAs are contained in exosomes and their molecular signature largely reflects that of the cells from which they are originated. Exosomes exist in the body fluids such as blood and urine and thus are expected to be a new marker for various diseases including cancer. Yoshioka et al. demonstrated that CD147 embedded in cancer-linked EV in blood can be used for detection of colorectal cancer [2]. Melo et al. recently reported that exosomes expressing glypican-1 in blood can differentiate patients with pancreatic cancer from healthy subjects and those with benign pancreatic disease [3].

Prostate cancer (PC), one of the most common male cancer, is the second-leading cause of cancer death among men in the United States [4]. PC well responds to androgen deprivation therapy, but 10 to $20 \%$ of patients develop castration-resistant prostate cancer (CRPC) [5]. In patients with advanced CRPC, bone metastasis is commonly found. Docetaxel, a microtubule-stabilizing taxane, has been used as the first-line chemotherapy for CRPC, but there is a finite amount of time before acquiring resistance $[6,7]$. The recent introduction of cabazitaxel, enzalutamide and abiraterone has expanded treatment options for metastatic CRPC patients [8].

Prostate-specific antigen (PSA) has been commonly used as a marker for $\mathrm{PC}$, but it cannot differentiate PC from benign prostatic hyperplasia $(\mathrm{BPH})$ unless $\mathrm{PC}$ is advanced and shows much higher serum PSA levels than $\mathrm{BPH}[9,10]$. In conjunction with measuring PSA levels, imaging modalities such as CT, MRI and bone scan are recommended to monitor the status of patients. There are numerous reports that identified potential markers to diagnose $\mathrm{PC}$, to diagnose progression or aggressiveness of CRPC and to predict prognosis of PC [11]. Since serial prostate biopsy is not usually performed due to its invasiveness and inaccuracy, it would be of great benefit if PC could be diagnosed and monitored by exosomes in the body fluids. We and others have demonstrated that prostate-specific membrane antigen (PSMA) and P-glycoprotein (P-gp) encoded by multidrug resistance protein 1 (MDR1) expressed on the surface of blood exosomes could be a marker for PC and taxane-resistant CRPC, respectively [12-15]. We have also recently reported the potential of integrin $\beta 4$ and vinculin in exosomes as markers for progression and aggressiveness of CRPC [16].

In the present study, we aimed to identify novel exosomal markers for PC especially those for castration-resistance and bone metastasis by analyzing exosomes secreted from PC cell lines including androgen-dependent $\mathrm{LNCaP}$ cell line and its sublines of partially androgen-independent $\mathrm{C} 4$, androgen-independent C4-2 and bone metastatic C4-2B [17, 18]. Among proteins identified by proteomic analysis, we focused on gamma-glutamyltransferase 1 (GGT1), a cell-surface enzyme that regulates the catabolism of extracellular L-gamma-glutamyl-L-cysteinylglycine (glutathione; GSH). Since GGT activity correlated with GGT1 expression in serum exosomes isolated by differential centrifugation, we measured GGT activity in patients. Contrary to our expectation, we found that serum exosomal GGT activity was significantly higher in PC patients than in BPH patients, which was supported by the finding that GGT1 expression was increased in PC tissues compared with BPH tissues. Altogether, we have identified serum exosomal GGT activity as a novel marker to diagnose $\mathrm{PC}$ or to distinguish $\mathrm{PC}$ from $\mathrm{BPH}$.

\section{Methods \\ Cell culture}

Human prostate cancer $\mathrm{LNCaP}$ cell line and its sublines of C4, C4-2 and C4-2B cell lines were obtained from the MD Anderson Cancer Center (Houston, TX, USA) and cultured in DMEM/Ham's F12 (4:1) medium supplemented with $10 \%$ fetal bovine serum, $5 \mu \mathrm{g} / \mathrm{mL}$ insulin, $13.65 \mathrm{pg} / \mathrm{mL}$ triiodo-thyronine, $4.4 \mu \mathrm{g} / \mathrm{mL}$ apotransferrin, $0.244 \mu \mathrm{g} / \mathrm{mL}$ d-biotin and $12.5 \mu \mathrm{g} / \mathrm{mL}$ adenine in a humidified atmosphere containing $5 \% \mathrm{CO}_{2}$.

\section{Isolation of exosomes by differential centrifugation}

Cells $\left(3.5 \times 10^{6}\right)$ seeded on $150-\mathrm{mm}$ dish were cultured for $72 \mathrm{~h}$ in DMEM/Ham's F12 (4:1) medium containing $10 \%$ exosome-deprived fetal bovine serum and other supplements described above. Exosomes were isolated from the conditioned medium as previously described [19]. Briefly, the medium was centrifuged at $2000 \mathrm{xg}$ for $10 \mathrm{~min}$ to eliminate cells. Second, the supernatant was centrifuged at $12000 \mathrm{xg}$ for $30 \mathrm{~min}$ to remove debris. Third, the supernatant was filtered through $0.22 \mu \mathrm{m}$ polyvinylidene difluoride (PVDF) filter. Finally, exosomes were pelleted by ultracentrifugation at $110,000 \mathrm{xg}$ for $70 \mathrm{~min}$, resuspended in $\mathrm{PBS}$ and stored at $-80{ }^{\circ} \mathrm{C}$ until use.

\section{Isolation of exosomes by immunocapture}

Mouse monoclonal anti-CD9 antibody (BioLegend, San Diego, CA, USA) and anti-PSMA antibody (MBL, Nagoya, Japan) were conjugated with Dynabeads M-270 epoxy magnetic beads (Life Technologies, Eugene, OR, USA) according to the manufacturer's protocol. The conditioned medium was centrifuged at $2000 \mathrm{xg}$ for $10 \mathrm{~min}$ and the supernatant was centrifuged at $12000 \mathrm{xg}$ for $30 \mathrm{~min}$. The supernatant was filtered through $0.22 \mu \mathrm{m}$ PVDF filter and $30 \mathrm{~mL}$ of the filtrate were 
incubated with $1 \mathrm{mg}$ of the antibody-conjugated beads at $4{ }^{\circ} \mathrm{C}$ for $90 \mathrm{~min}$ with rotation. The beads were washed 3 times with PBS and resuspended in sample buffer. After separation from magnetic beads, samples were boiled and stored at $-20{ }^{\circ} \mathrm{C}$ until use.

\section{Isolation of exosomes by size exclusion chromatography}

A commercially available size exclusion chromatography column, EVSecond (GL Science, Tokyo, Japan), was used for isolation of exosomes. After washing with PBS, $500 \mu \mathrm{L}$ serum was loaded onto the column and eluted with PBS. The first $1 \mathrm{~mL}$ of eluate was discarded and thereafter the eluate was collected in 24 fractions of $0.1 \mathrm{~mL}$ each.

\section{Quantitative proteomic analysis}

Proteomic analysis was performed as previously described [16]. In brief, exosomes were labeled with iTRAQ reagents using the iTRAQ multiplex kit (AB Sciex, Foster City, CA, USA). Labeled samples were separated and automatically spotted onto a MALDI plate using the direct nanoLC and MALDI fraction system DiNaMaP (KYA Technologies, Tokyo Japan). Mass spectra were acquired using the $\mathrm{AB}$ Sciex TOF/TOF 5800 system operated on the TOF/TOF Series Explorer software version 4.1 (AB Sciex). All MS/MS data were submitted to the ProteinPilot software version 4.5 (AB Sciex). Protein identification was considered to be correct based on the following selection criteria: protein having at least 2 peptides with an ion score above $95 \%$ confidence; and protein with protein score (ProtScore) $>1.3$ (unused, $p<0.05,95 \%$ confidence).

\section{Western blot analysis}

Whole cell lysates were prepared in ice-cold lysis buffer (1\% Igepal CA-630, 1\% sodium deoxycholate, 0.1\% SDS, $150 \mathrm{mM} \mathrm{NaCl}, 25 \mathrm{mM}$ Tris- $\mathrm{HCl}$ [pH 7.6]) containing protease inhibitor cocktail. Cell lysates and exosomes were subjected to electrophoresis on SDS-polyacrylamide gels and transferred to PVDF membranes. After blocking in $5 \%$ skim milk, membranes were hybridized with a primary antibody and then with a horseradish peroxidase-linked secondary antibody. After washing, bound proteins were visualized using the ECL Prime Western blotting detection system (GE Healthcare, Little Chalfont, UK) or Immunostar LD (Wako Pure Chemical Industries, Osaka, Japan). Anti-CD9, -PSMA and - $\beta$-actin antibodies were obtained from Cell Signaling Technology (Danvers, MA, USA). Antibodies recognizing GGT1 small subunit was purchased from Abnova (Taipei, Taiwan). Anti-GGT1 large subunit and -Alix antibody were from Santa Cruz Biotechnology (Santa Cruz, CA, USA).

\section{Measurement of CD9 level}

The CD9 level in exosomes was determined by a sandwich ELISA. A MaxiSorp micro titer plate (Thermo Fisher Scientific, MA, USA) was coated with $5 \mu \mathrm{g} / \mathrm{mL}$ anti-CD9 antibody (Ancell Corporation, Bayport, MN, USA) in carbonate buffer ( $\mathrm{pH}$ 9.6) at $4{ }^{\circ} \mathrm{C}$ overnight. After washing 3 times with PBS, $200 \mu \mathrm{L}$ of $1 \%$ BSA/PBS was added and incubated at room temperature for $1 \mathrm{~h}$ with shaking. After washing, sample was added in a final volume of $100 \mu \mathrm{L}$ and incubated at room temperature for $2 \mathrm{~h}$. After washing, $0.5 \mu \mathrm{g} / \mathrm{mL}$ biotinylated anti-CD9 antibody (Ancell Corporation) in 1\% BSA/PBS was added in a final volume of $100 \mu \mathrm{L}$ and incubated at room temperature for $1 \mathrm{~h}$. After washing, 1:5000 diluted streptavidin-AP (Roche, Basel, Switzerland) in 1\% BSA/PBS was added in a final volume of $100 \mu \mathrm{L}$ and incubated at room temperature for $1 \mathrm{~h}$. After washing 6 times with PBS, CDP-Star substrate with Emerald II Enhancer (Thermo Fisher Scientific) was added and chemiluminescence was recorded by the EnVision Multilabel Reader (PerkinElmer, MA, USA).

\section{Measurement of GGT activity}

GGT activity was measured using a fluorescent probe, $\gamma$-glutamyl hydroxymethyl rhodamine green (gGluHMRG), which is commercially called ProteoGREENgGlu (Goryo Chemical, Hokkaido, Japan) [20]. Twenty microliter of sample was reacted with $180 \mu \mathrm{L}$ of $1.11 \mu \mathrm{M}$ ProteoGREEN-gGlu in PBS in each well of 96-well black plates (Corning, NY, USA). The plate was incubated at room temperature for $1 \mathrm{~h}$ and fluorescence intensity (Ex/Em 490/520 nm) was measured using the EnVision Multilabel Reader (PerkinElmer).

\section{OptiPrep density gradient centrifugation}

Five hundred microliter of serum was centrifuged at $12000 \mathrm{xg}$ for $30 \mathrm{~min}$ and the supernatant was filtered through $0.22 \mu \mathrm{m}$ PVDF filter. The filtered sample was diluted with $11 \mathrm{~mL}$ of PBS and centrifuged at 110,000 $\mathrm{xg}$ for $70 \mathrm{~min}$. The pellet was resuspended in $500 \mu \mathrm{L}$ of PBS. A stock solution of OptiPrep $(60 \% \quad w / v$ iodixanol) (Axis-Shield, Dundee, Scotland) was diluted with $0.25 \mathrm{M}$ sucrose, $10 \mathrm{mM}$ Tris- $\mathrm{HCl}(\mathrm{pH}$ 7.6) to generate $40 \%, 20 \%, 10 \%$ and $5 \% w / v$ iodixanol solutions. A discontinuous density gradient was generated by sequential layering of $3 \mathrm{~mL}$ each of 40 , 20 and $10 \%(w / v)$ iodixanol solutions, followed by $2.5 \mathrm{~mL}$ of $5 \%$ iodixanol solution in ultracentrifuge tubes. Sample was overlaid on the discontinuous iodixanol gradient followed by centrifugation at $110,000 \mathrm{xg}$ for $16 \mathrm{~h}$. One milliliter fractions were collected from the top of the gradient. Each sample 
was diluted with $11 \mathrm{~mL}$ of PBS and centrifuged at $110,000 \mathrm{xg}$ for $70 \mathrm{~min}$. The pellet was resuspended in PBS and stored at $4{ }^{\circ} \mathrm{C}$ until use.

\section{Collection of blood from patients and isolation of exosomes}

This study was approved by the Bioethics Committees of Gifu University and Tokyo Metropolitan Institute of Gerontology and a written informed consent was obtained from all patients. Thirty-nine patients suspicious of PC due to either abnormal MRI findings or elevated PSA levels were recruited. Blood was corrected from patients prior to biopsy. After biopsy, 31 patients and 8 patients were pathologically diagnosed as $\mathrm{PC}$ and $\mathrm{BPH}$, respectively. Serum was separated from whole blood by centrifugation at $1800 \mathrm{xg}$ and stored at $-80{ }^{\circ} \mathrm{C}$ until use. For exosome isolation, $210 \mu \mathrm{L}$ of serum was centrifuged at $12000 \mathrm{xg}$ for $30 \mathrm{~min}$ and the supernatant was filtered through $0.22 \mu \mathrm{m}$ PVDF filter. The $200 \mu \mathrm{L}$ of filtered sample diluted with $800 \mu \mathrm{L}$ of PBS was centrifuged at 100,000 xg for $75 \mathrm{~min}$. The pellet was washed in PBS and centrifuged at 100,000 xg for $75 \mathrm{~min}$. The final pellet was resuspended in PBS and stored at $4{ }^{\circ} \mathrm{C}$ until use.

\section{Immunohistochemical analysis of GGT1}

This study was approved by the Bioethics Committees of Tokyo Metropolitan Institute of Gerontology. Formalinfixed paraffin-embedded biopsies and surgically resected tissue specimens from PC $(n=50)$ and BPH $(n=50)$ patients were stained for GGT1. The tissue sections $(3 \mu \mathrm{m})$ were subjected to immunostaining using antiGGT1 antibody raised against the small subunit (Abnova). After deparaffinization, the sections were preheated in Heat processor solution $(\mathrm{pH}$ 6.0, Nichirei, Tokyo, Japan) at $100{ }^{\circ} \mathrm{C}$ for $30 \mathrm{~min}$. The sections were then incubated with the anti-GGT1 antibody (1:800 in dilution) at $4{ }^{\circ} \mathrm{C}$ overnight. Bound antibodies were detected with the Envision kit (Dako Denmark A/S, Glostrup, Denmark) using diaminobenzidine tetrahydrochloride as a substrate. The sections were then counterstained with Mayer's hematoxylin. Negative control tissue sections were prepared by omitting the primary antibody. In order to evaluate GGT1 expression, the intensity $(1,0+; 2,1+; 3,2+; 4,3+)$ and percentage $(1,0-25 \% ; 2,26-50 \% ; 3,51-75 \% ; 4,76-100 \%)$ of membranous and cytoplasmic GGT1 staining were scored. GGT1 expression in the prostatic glands and prostatic cancer cells was evaluated under $\times 200$ magnification. The score of intensity multiplied by that of percentage was used as the final score for GGT1 expression. Two independent pathologists blinded to the clinical and pathological information performed scoring.

\section{Statistical analysis}

Statistical differences were determined by one-way ANOVA with Tukey's multiple comparison tests (for comparison among cell lysates and exosomes isolated from cultured cells), Welch's t-test (for comparison among serum PSA concentration, serum GGT activity and serum exosomal GGT activity), Brunner-Munzel test (for comparison between $\mathrm{BPH}$ and $\mathrm{PC}$ in immunohistochemical analysis) or paired Student's t-test (for comparison between cancerous and non-cancerous lesions in immunohistochemical analysis). Spearman's rank correlation coefficient was used to evaluate the correlation between GGT activity and GGT1 expression. $p<0.05$ was considered statistically significant.

\section{Results}

Identification of GGT1 as a potential exosomal marker for PC based on proteomic analysis of exosomes isolated from PC cells by differential centrifugation

We analyzed androgen-dependent $\mathrm{LNCaP}$ cell line and its sublines of C4, C4-2 and C4-2B cell lines $[17,18]$. The $\mathrm{C} 4$ cell was established from $\mathrm{LNCaP}$ cell transplantation under castration and showed low sensitivity to androgen. The C4-2 cell was established from C4 cell transplantation under long term castrated condition and showed androgen independent growth response. The $\mathrm{C} 4-2 \mathrm{~B}$ cell was established from bone metastasis of C4-2 cell transplantation under castration. Exosomes were isolated from the cell culture medium by differential centrifugation. In order to identify differentially expressed proteins in exosomes, we performed iTRAQ-based quantitative proteomic analysis of exosomes. A total of 153 proteins were detected (Additional file 1: Table S1) and eight proteins were found to be upregulated by more than 1.5 -fold in exosomes isolated from $\mathrm{C} 4-2 \mathrm{~B}$ cells compared with those from parental LNCaP cells (Table 1). Among them was GGT1, a cell-surface enzyme that cleaves extracellular GSH and provides cells with amino acids, thereby increasing the intracellular GSH level [21]. GGT1 was upregulated in C4-2 cells (1.56-fold) as well as in C4-2B cells (1.63-fold). Since serum GGT activity is reported to be elevated in patients with certain types of cancer [22], we focused on GGT1 as a potential exosomal marker for PC in the subsequent studies.

\section{Upregulation of GGT1 expression in exosomes isolated from C4-2 and C4-2B cells by differential centrifugation} GGT1 is comprised of a large subunit that anchors the enzyme to the cell membrane and a small subunit that binds to and catalyzes the first step in the degradation of extracellular GSH [23]. Western blot analysis showed elevation of GGT1 large and small subunits in exosomes isolated from $\mathrm{C} 4-2$ and $\mathrm{C} 4-2 \mathrm{~B}$ cells compared with LNCaP and C4 cells (Fig. 1a). 
Table 1 Proteins upregulated (>1.5 fold) in C4-2B exosomes compared with LNCaP exosomes

\begin{tabular}{llllll}
\hline Accession number & Protein name & Gene symbol & C4/LNCaP & C4-2/LNCaP & C4-2B/LNCaP \\
\hline P05106 & Integrin beta-3 & ITGB3 & 0.78 & 1.50 & 2.00 \\
O60716 & Catenin delta-1 & CTNND1 & 1.10 & 1.58 & 1.94 \\
P35221 & Catenin alpha-1 & CTNNA1 & 0.97 & 1.51 & 1.19 \\
P02765 & Alpha-2-HS-glycoprotein & AHSG & 0.96 & 0.82 & 1.56 \\
P19440 & Gamma-glutamyltranspeptidase 1 & GGT1 & 0.75 & 1.25 & 1.71 \\
P61224 & Ras-related protein Rap-1b & RAP1B & 1.66 & 1.29 & 1.58 \\
P62258 & 14-3-3 protein epsilon & YWHAE & 1.13 & 1.56 & 1.54 \\
P53985 & Monocarboxylate transporter 1 & SLC16A1 & & 1.51 \\
\hline
\end{tabular}

PSMA as well as CD9, an exosomal marker, were detected in exosomes isolated from four cell lines. Consistent with increased expression in exosomes, both GGT1 large and small subunits were upregulated in C4-2 and C4-2B cells, whereas the PSMA expression level was similar among 4 cell lines (Fig. 1b). These results confirmed that GGT1 expression in exosomes was increased in castration-resistant $\mathrm{C}_{4}-2$ and bone metastatic C4-2B cell lines.

\section{Upregulation of GGT1 expression in exosomes isolated} from C4-2 and C4-2B cells by immunocapture

When exosomes were isolated from the cell culture medium by the immunocapture method using anti-CD9

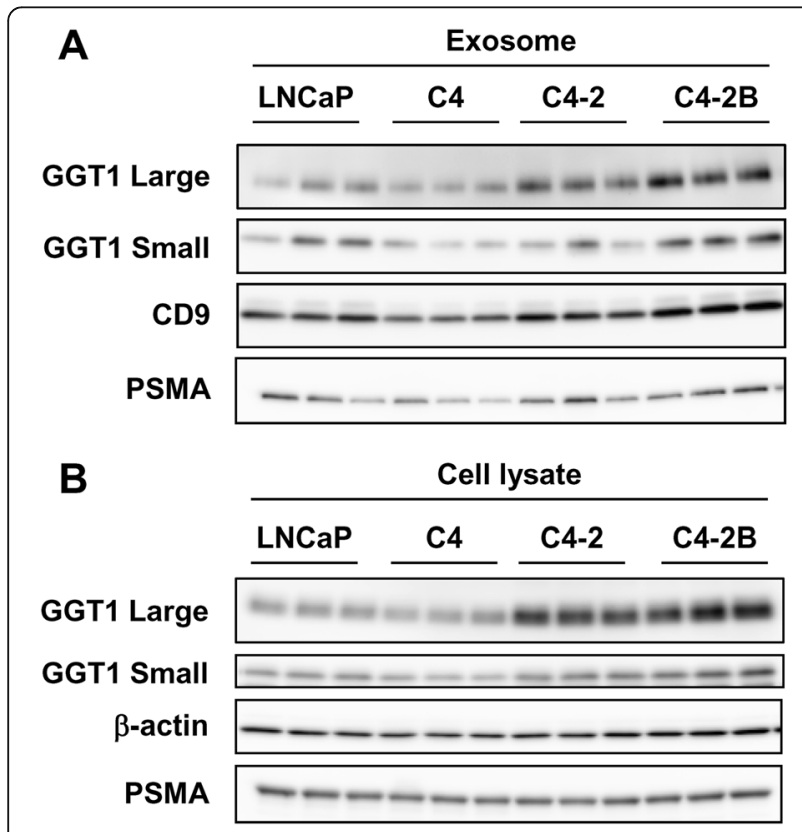

Fig. 1 GGT1 expression in exosomes isolated from PC cells by differential centrifugation. Exosomes were isolated from the culture medium of LNCaP, C4, C4-2 and C4-2B cells by differential centrifugation. Exosomes (a) and cell lysates (b) were subjected to Western blot analysis for GGT1 large and small subunits, CD9, PSMA and $\beta$-actin antibody, the levels of GGT1 large and small subunits were elevated in C4-2 and C4-2B cells, while expression of PSMA as well as exosomal markers, CD9 and Alix, showed no major difference among cell lines (Fig. 2a). Similarly, GGT1 large and small subunits were upregulated in exosomes captured from C4-2 and C4-2B cells by anti-PSMA antibody (Fig. 2b). These results suggested that distinct subsets of exosomes positive for CD9 or PSMA exhibited increased expression of GGT1.

\section{Correlation of GGT activity with GGT1 expression in exosomes isolated from PC cells}

Here we measured GGT activity in exosomes using a fluorescence imaging probe, $\gamma$-glutamyl hydroxymethyl

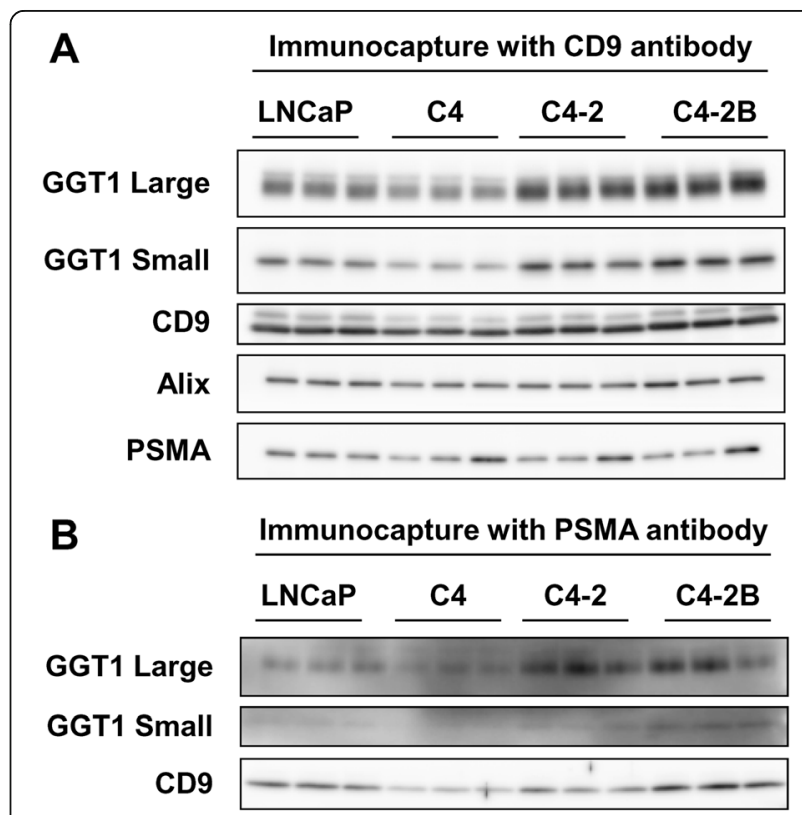

Fig. 2 GGT1 expression in exosomes isolated from PC cells by immunocapture. The culture medium of LNCaP, C4, C4-2 and C4-2B cells $(30 \mathrm{~mL})$ were incubated with magnetic beads (1 mg) conjugated with anti-CD9 (a) or -PSMA (b) antibody at $4{ }^{\circ} \mathrm{C}$ for 90 min. Whole immunocaptured exosomes were subjected to Western blot analysis for GGT1 large and small subunits, CD9, Alix and PSMA 
rhodamine green (gGlu-HMRG) that is activated by cleavage of glutamate with GGT [20]. GGT activity was measurable in both cell lysates and exosomes isolated from $\mathrm{LNCaP}, \mathrm{C} 4, \mathrm{C} 4-2$ and $\mathrm{C} 4-2 \mathrm{~B}$ cells. There was a significant increase in GGT activity in C4-2B cells compared with LNCaP cells (Fig. 3a). On the other hand, GGT activities were higher in exosomes isolated from C4-2 and C4-2B cells than in those from LNCaP and C4 cells (Fig. 3b). More importantly, GGT activity in exosomes correlated with the expression levels of GGT1 large and small subunits in exosomes among 4 cell lines (Fig. 1a). These results indicated that exosomal GGT activity could be used as an alternative to exosomal GGT1 expression.

\section{GGT activity and GGT1 expression in exosomes isolated} from human serum

Franzini et al. identified four GGT fractions in serum: big-GGT, medium-GGT, small-GGT and free-GGT fractions [24] and recently showed that the big-GGT fraction corresponds to exosomal GGT [25]. Here we subjected serum of a healthy individual to size exclusion chromatography (SEC) and measured GGT activity and CD9 expression in each fraction. The level of CD9 was determined by a sandwich ELISA. SEC yielded a minor peak and a major peak of GGT activity (Fig. 4a). The minor peak spanning fractions 4 to 9 was positive for CD9, indicating that GGT activity was detected in serum exosomes. Western blot analysis of the fractions 3 to 10 obtained from the same healthy individual revealed the co-presence of GGT1 large and small subunits with CD9 (Fig. 4b). We also subjected exosomes isolated from human serum by differential centrifugation to OptiPrep density gradient centrifugation. The results showed that GGT1 large and small subunits were detected only in fraction 9 that was positive for CD9 (Fig. 5a), indicating that serum exosomes isolated by differential centrifugation is free of contamination with other GGT forms such as medium-GGT, small-GGT and free-GGT. Lastly, we

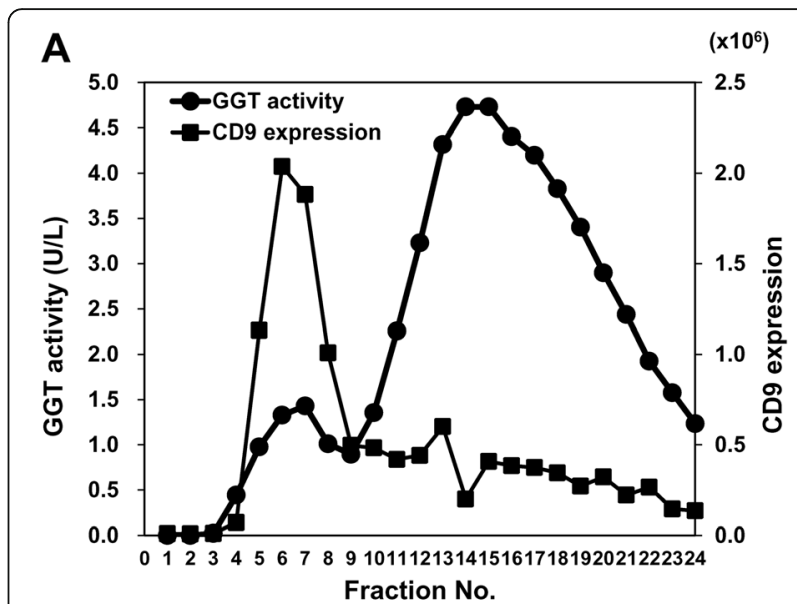

B $\quad$ Fraction No. $\begin{array}{lllllllll}3 & 4 & 5 & 6 & 7 & 8 & 9 & 10\end{array}$

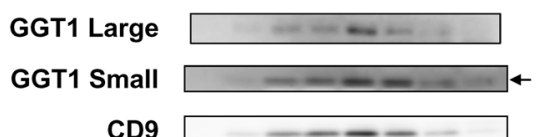

Fig. 4 GGT activity and GGT1 expression in exosomes isolated from human serum by SEC. Serum of a healthy individual $(500 \mu \mathrm{L})$ was subjected to SEC. a CD9 expression in each fraction was measured by a sandwich ELISA. GGT activity in each fraction was determined by incubation with gGlu-HMRG at room temperature for $1 \mathrm{~h}$ and measurement of fluorescence intensity (Ex/Em 490/520 nm) using microplate reader. $\mathbf{b}$ The fractions 3-10 collected from a healthy individual were subjected to Western blot analysis for GGT1 large and small subunits and CD9. The upper band of the doublet corresponds to the GGT1 small subunit (shown by arrow)

isolated serum exosomes by differential centrifugation from $\mathrm{BPH}(n=4)$ and PC $(n=8)$ patients and determined GGT1 expression (Fig. 5b) as well as GGT activity. Spearman's rank correlation analysis revealed correlation of GGT activity with the signal intensity of GGT1 large subunit in serum exosomes (Fig. 5c). These results provided the basis for measuring GGT activity in serum exosomes isolated by differential centrifugation using the gGlu-HMRG probe.
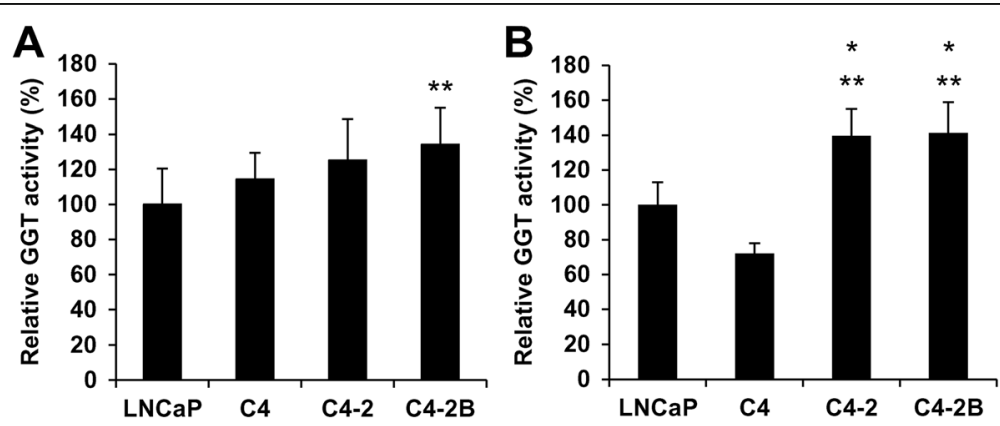

Fig. 3 GGT activity in cell lysates and exosomes isolated from PC cells by differential centrifugation. Cell lysates (a) and exosomes isolated from the culture medium of $L N C a P, C 4, C 4-2$ and C4-2B cells by differential centrifugation (b) were mixed with gGlu-HMRG. After incubation at room temperature for $1 \mathrm{~h}$, fluorescence intensity (Ex/Em 490/520 nm) was measured by using microplate reader. GGT activity is shown as a percentage of LNCaP cells. ${ }^{*} p<0.05$, compared with $L N C a P$ cells, ${ }^{* *} p<0.01$, compared with C4 cells 


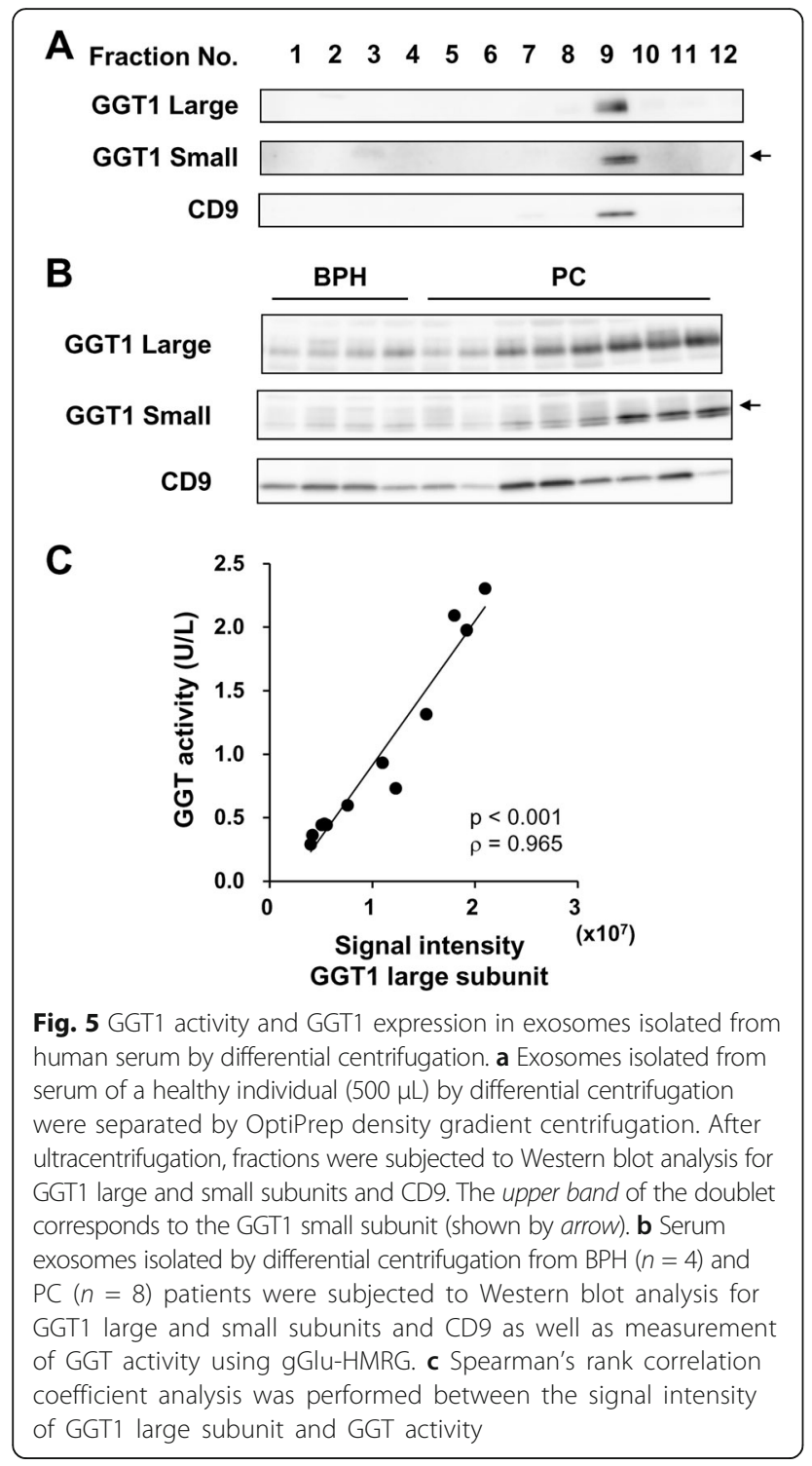

No association between serum exosomal GGT activity and CRPC

Since we identified GGT1 as an exosomal marker upregulated in castration-resistant C4-2 and bone metastatic C4-2B cells, we hypothesized that GGT activity in serum exosomes could be a marker for CRPC and/or bone metastasis. We isolated exosomes by differential centrifugation from serum of patients with $\mathrm{PC}$ and measured GGT activity using the gGlu-HMRG probe. Contrary to our expectation, however, serum exosomal GGT activity exhibited no difference between PC patients with $(n=6$, PSA: $7.46-585.70 \mathrm{ng} / \mathrm{mL})$ and without ( $n=35$, PSA: $4.20-549.39 \mathrm{ng} / \mathrm{mL}$ ) castrationresistance (Additional file 2: Fig. S1). The association of serum exosomal GGT activity with bone metastasis was not examined due to limited number of appropriate patients. These results suggested that GGT activity in serum exosomes isolated by differential centrifugation would have little or no potential as a marker for CRPC in $\mathrm{PC}$ patients.

\section{Increased serum exosomal GGT activity in PC patients than in BPH patients}

It has been reported that serum GGT activity was increased in certain types of cancer [22] and thus we measured serum GGT activity as well as serum exosomal GGT activity in patients with $\mathrm{BPH}(n=8$, PSA: 4.42-25.40 ng/mL) and PC patients $(n=31$, PSA: 4.20-28.23 ng/mL). The results showed that there was no statistical difference in the serum PSA concentration (Fig. 6a) and serum GGT activity (Fig. 6b) between two patient groups. In contrast, GGT activity in serum exosomes was significantly increased in

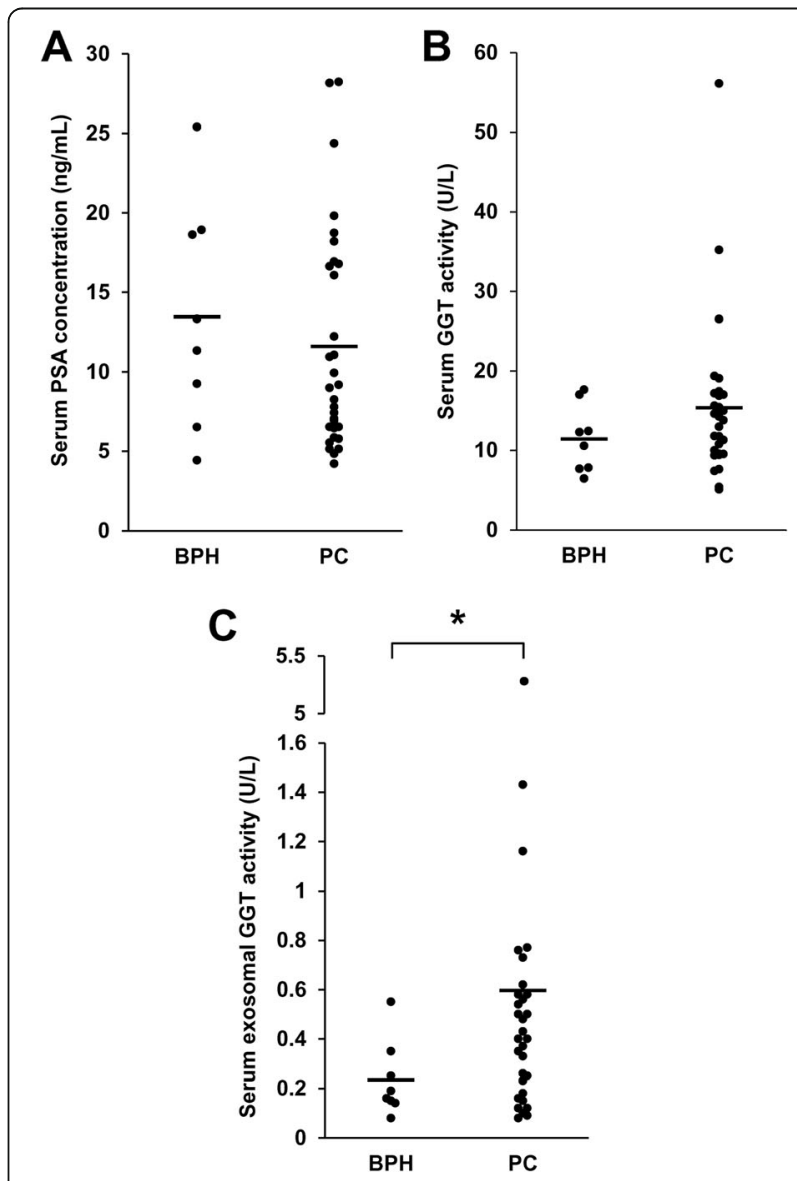

Fig. 6 GGT activity in exosomes isolated by differential centrifugation from serum of PC and BPH patients. Exosomes were isolated by differential centrifugation from serum $(210 \mu \mathrm{L})$ of PC $(n=31)$ and BPH $(n=8)$ patients. GGT activity was determined by incubation with gGlu-HMRG at room temperature for $1 \mathrm{~h}$ and measurement of fluorescence intensity (Ex/Em 490/520 nm) using microplate reader. Patient groups were compared for serum PSA concentration (a), serum GGT activity (b) and serum exosomal GGT activity (c). ${ }^{*} p<0.05$, compared with $\mathrm{BPH}$ patients 
patients with PC compared to those with BPH (Fig. 6c). These results suggested that serum exosomal GGT activity but not serum GGT activity could be a biomarker to distinguish PC patients from $\mathrm{BPH}$ patients, both of which exhibited similar serum PSA levels.

\section{Increased GGT1 expression in PC tissues than in BPH tissues}

Elevated serum exosomal GGT activity in PC patients compared with $\mathrm{BPH}$ patients suggested the possibility that GGT1 expression might be increased in PC tissues than in BPH tissues. In order to prove our hypothesis, we performed immunohistochemical staining of GGT1 using formalin-fixed paraffin-embedded biopsies and surgically resected tissue specimens from $\mathrm{PC}$ and $\mathrm{BPH}$ patients. The clinical and pathological profile of patients is shown in Additional file 3: Table S2. In BPH tissues, prostatic glands showed weak apical expression for GGT1 (Fig. 7a). In PC tissues, cancer cells showed cytoplasmic and membranous expression for GGT1 and background noncancerous prostatic glands showed weak apical expression. In order to evaluate GGT1 expression on the plasma membrane and in the cytoplasm, staining was scored for the intensity and percentage and then both scores were multiplied (Fig. 7b). GGT1 expression on the plasma membrane was increased in PC tissues compared with $\mathrm{BPH}$ tissues $(p<0.01)$, whereas that in the cytoplasm showed no statistically significant difference. When GGT1 expression was compared within the PC tissues, membranous and cytoplasmic expression was higher in the cancerous lesion than in the noncancerous lesion $(p<0.001$ and $p<0.001$, respectively). There were no statistical differences between GGT1 expression and Gleason score. These results indicated that GGT1 expression was elevated in PC tissues than in $\mathrm{BPH}$ tissues, supporting our findings of increased serum exosomal GGT activity in PC patients.

\section{Discussion}

Based on proteomic analysis of exosomes isolated from PC cell lines by differential centrifugation, we identified GGT1 as a potential exosomal marker for PC. GGT also known as gamma-glutamyl transpeptidase is an enzyme that transfers a gamma-glutamyl group from GSH and other $\gamma$-glutamyl compounds to amino acids or dipeptides. GSH is abundant in the cells and plays important roles in protection from oxidative stress and maintenance of the redox status [26]. GGT initiates the degradation of extracellular GSH, resulting in production of cysteinylglycine and glutamate. Cysteinylglycine is then hydrolyzed by cell surface dipeptidase to generate glycine and cysteine. The degraded amino acids are used for de novo synthesis of GSH. In normal human tissues, strong GGT immunoreactivity was observed on the

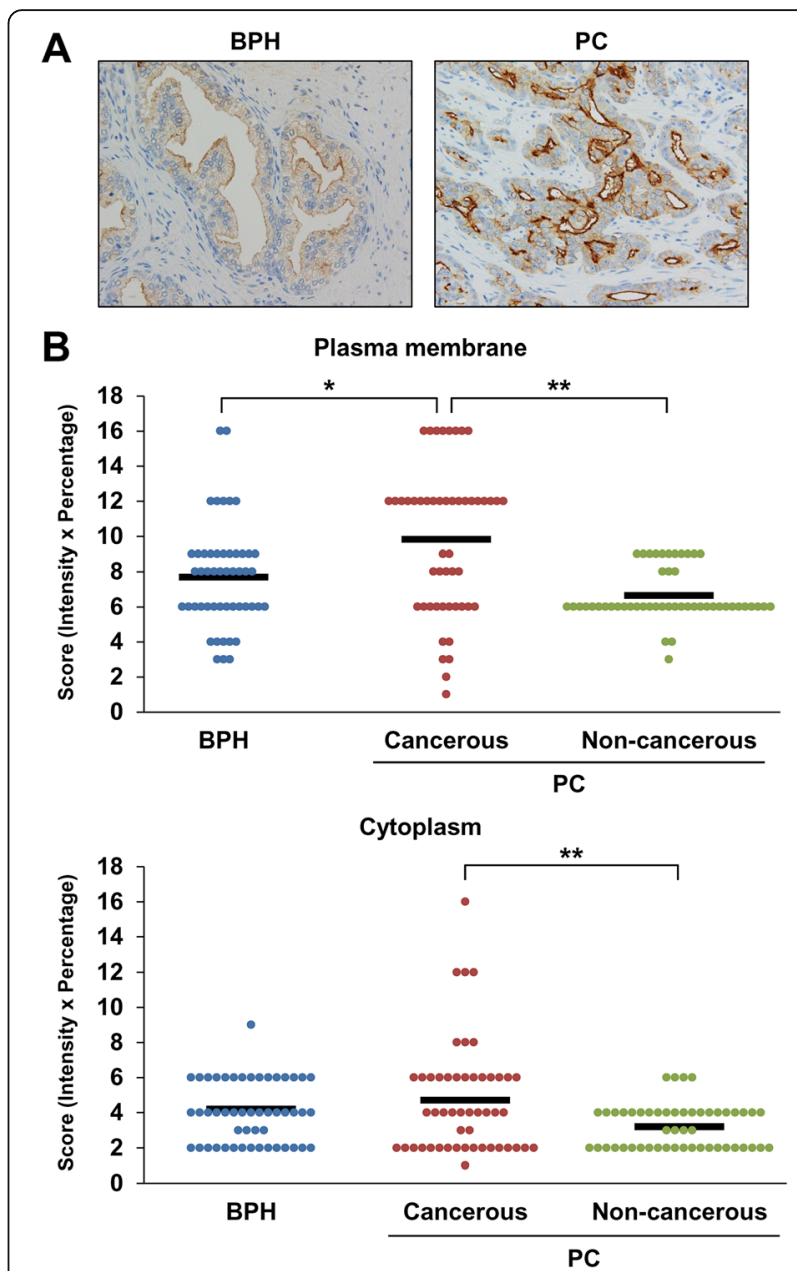

Fig. 7 Immunohistochemical analysis of GGT1 in PC and BPH tissues. Formalin-fixed paraffin-embedded biopsies and surgically resected tissue specimens from PC $(n=50)$ and BPH $(n=50)$ patients were stained for GGT1. a Representative images in BPH and PC tissues are shown. Original magnification $\times 400$. $\mathbf{b}$ GGT1 expression on the plasma membrane and in the cytoplasm of cancerous and non-cancerous lesions of PC and BPH tissues is expressed as a score calculated by multiplying the intensity score with the percentage score. ${ }^{*} p<0.01$, compared with $\mathrm{BPH}$ tissues. ${ }^{* *} p<0.001$, compared with the non-cancerous lesion

surface of renal proximal tubule cells, hepatic bile canaliculi and capillary endothelial cells within the nervous system [27]. Secretory or absorptive cells in sweat glands, prostate, salivary gland ducts, bile ducts, pancreatic acini, intestinal crypts and testicular tubules were also GGT-positive. Among a family of GGT genes in the human genome [28], GGT1, which is generally referred to as GGT, is shown to be involved in GSH metabolism [29].

Elevation of GGT expression has been reported for a number of cancers including colon, ovary and liver cancer, astrocytic glioma, soft tissue sarcoma, melanoma and leukemia [22]. A comprehensive analysis of GGT 
expression showed that most tumors derived from tissues expressing GGT were positive for GGT and that lung and ovary cancer derived from GGT-negative epithelia also expressed GGT [22]. GGT expression was linked to unfavorable prognostic signs in breast cancer, but no correlation between GGT expression and standard clinical pathological parameters has been found in prostatic, colorectal and breast cancer [22].

Upregulation of GGT expression in cancer has been considered to protect cancer cells against oxidative stress by increasing the intracellular GSH level and thereby support their growth and survival [30]. However, it was also demonstrated that the metabolism of GSH by GGT can exert pro-oxidant effects [31]. Upregulation of GGT may impose an increased oxidative burden on the cell, resulting in GSH consumption and a decrease of cellular GSH stores. The persistent production of ROS caused by increased GGT expression may contribute to genetic instability and tumor progression [32].

Serum GGT activity is commonly used as a marker for liver, gallbladder and biliary tract diseases especially alcoholic liver disease because it is particularly sensitive to alcohol consumption [33]. On the other hand, a positive association of serum GGT activity with the risk of cancer [34, 35] as well as cardiovascular diseases and metabolic syndrome [36] has been reported. Furthermore, serum GGT levels were found to be higher in hepatocellular carcinoma patients with poorly differentiated tumors as compared to those with well and moderately differentiated tumors [37]. In renal cell carcinoma, serum GGT activity was reported to be increased in most of patients with metastasis, while it was normal in majority of patients with localized tumor [38].

Franzini et al. performed gel filtration chromatography followed by postcolumn reaction with a fluorescent GGT substrate, gamma-glutamyl-7-amido-4-methylcoumarin ( $\gamma$ GluAMC) and identified four GGT fractions in serum: big-GGT, medium-GGT, small-GGT and freeGGT fractions of different molecular weight (molecular masses $>2000 \mathrm{kDa}, 940 \mathrm{kDa}, 140 \mathrm{kDa}$ and $70 \mathrm{kDa}$, respectively) [24]. The authors demonstrated that b-GGT increased in non-alcoholic fatty liver disease (NAFLD) but not in chronic hepatitis $\mathrm{C}(\mathrm{CHC})$ and that b-GGT/sGGT ratio showed the highest diagnostic accuracy for distinguishing NAFLD and CHC [39]. They also showed that the big-GGT fraction corresponds to serum exosomal GGT [25].

In order to determine GGT activity on exosomes, we used a newly reported fluorescence probe, gGlu-HMRG, which is activated by rapid one-step cleavage of glutamate with GGT [20]. This probe was developed to detect cancers cells during surgical and endoscopic procedures, taking advantage of its activation by GGT that is present on the cell surface. In vivo imaging of superficial head and neck squamous cell carcinoma and beast, lung and colorectal cancer using gGlu-HMRG has been reported [40-43]. In vitro activation of gGlu-HMRG was also shown in human ovarian cancer cell lines [20].

In the present study, we first showed correlation of GGT1 expression with GGT activity in cell lysates and exosomes. Second, we separated human serum by SEC and demonstrated that the minor peak that was positive for CD9 contained GGT1 large and small subunits as well as GGT activity and that the major peak was presumably comprised of medium-GGT, small-GGT and free-GGT fractions other than big-GGT or exosomal GGT fraction. Third, we subjected exosomes isolated from human serum by differential centrifugation to OptiPrep density gradient centrifugation and confirmed that exosomes isolated from human serum by differential centrifugation is free of contamination with other GGT forms. Lastly, based on these findings, we measured serum exosomal GGT activity in patients. Despite the fact that GGT1 was upregulated in exosomes isolated from androgen-independent $\mathrm{C} 4-2$ and bone metastatic $\mathrm{C} 4-2 \mathrm{~B}$ cells, there was no difference between PC patients with and without castrationresistance. Unexpectedly, we found that serum exosomal GGT activity was significantly higher in PC patients than in $\mathrm{BPH}$ patients.

In support of our findings of increased serum exosomal GGT activity in PC patients, GGT1 expression was elevated in $\mathrm{PC}$ tissues compared with $\mathrm{BPH}$ tissues. A previous report showed that the majority of neoplastic cells were positive for GGT1 in most of PC [44]. In the present study, we demonstrated that there was a significant difference in GGT1 expression between PC and $\mathrm{BPH}$ tissues. Furthermore, cancer cells showed stronger expression for GGT1 in the cytoplasm and membrane than background noncancerous prostatic glands. These results suggested that prostatic cancer cells may produce more exosomes expressing GGT1. The underlying mechanism that is responsible for overexpression of GGT1 in PC remains to be elucidated.

Numerous reports have proposed potential markers for PC based on pathological and clinical research [45]. More recently identified PC markers include prostate cancer antigen 3 (PCA3) [46], TMPRSS2ERG fusion gene [47] and their combined use [48]. Although there have been a limited number of reports describing exosomal miRNA as a marker for PC [49], we and others have reported exosomal protein markers that would be helpful to diagnose PC (PSMA), taxane-resistant CRPC (P-gp) and progression and aggressiveness of $\mathrm{PC}$ (integrin $\beta 4$ and vinculin) [12-16]. This is the first report that described serum exosomal GGT1 expression or GGT activity as a potential marker to diagnose PC. 
PSA is a commonly used marker for PC, but it cannot distinguish $\mathrm{PC}$ from $\mathrm{BPH}$ when the levels are similar $[9,10]$. In the present study, we measured serum exosomal GGT activity as well as serum GGT activity and serum PSA level in two patient groups. As shown in Additional file 4: Fig. S2, the AUC of serum exosomal GGT activity was 0.714 (95\% CI between 0.535 and 0.892 ), while that of serum GGT activity was 0.621 (95\% CI between 0.396 and 0.846 ) and that of serum PSA concentration was 0.601 (95\% CI between 0.361 and 0.841 ). These results suggest that serum exosomal GGT activity but not serum GGT activity could be a biomarker to differentiate PC patients from BPH patients, both of which exhibit similar serum PSA levels.

Although we have demonstrated the potential of serum exosomal GGT activity for differential diagnosis of $\mathrm{PC}$ and $\mathrm{BPH}$, the current detection system has limitations for clinical application, because differential centrifugation is required to measure the activity. It is also worth noting that GGT1 is expressed in normal tissues and thus serum exosomes isolated by differential centrifugation may contain those derived from various tissues. We and others have recently demonstrated that exosomes derived from PC could be isolated by immunocapture with anti-PSMA antibody $[12,13]$. The development of an antibody with a higher affinity for PSMA and its use would enable us to increase the specificity and sensitivity of serum exosomal GGT activity as a marker for PC.

The usefulness of serum exosomal GGT activity as a maker to diagnose PC needs to be validated in large-scale clinical studies. Since serum GGT activity has been implicated in a variety of diseases by clinical and epidemiological studies [34-36, 50], it would be of great interest to test if serum exosomal GGT activity is superior to serum GGT activity in other diseases than PC. Nevertheless, in order to conduct large-scale studies, a simple and rapid detection system remains to be established, which would make it possible to evaluate the potential of serum exosomal GGT activity as prognostic as well as diagnostic markers in prospective clinical studies. Finally, it is also of great importance to understand the properties and roles of GGT1 on exosomes in serum of patients.

\section{Conclusions}

We demonstrated that GGT activity in serum exosomes was significantly higher in PC patients than in $\mathrm{BPH}$ patients, which was supported by increased GGT1 expression in PC tissues compared with $\mathrm{BPH}$ tissues. Serum exosomal GGT activity could be a useful marker to diagnose $\mathrm{PC}$ or to distinguish PC from $\mathrm{BPH}$ and possibly to diagnose other types of cancer with increased GGT1 expression.

\section{Additional files}

Additional file 1: Table S1. List of differentially expressed proteins. (PDF $97 \mathrm{~kb}$ )

Additional file 2: Fig. S1. GGT activity in exosomes isolated by differentia centrifugation from serum of PC patients. (PDF $40 \mathrm{~kb}$ )

Additional file 3: Table S2. Patient characteristics. (PDF $77 \mathrm{~kb}$ ) Additional file 4: Fig. S2. $R O C$ curve analysis of $P C$ and BPH patients. (PDF $30 \mathrm{~kb}$ )

\section{Abbreviations}

$\mathrm{BPH}$ : Benign prostatic hyperplasia; $\mathrm{CHC}$ : Chronic hepatitis C; CRPC: Castrationresistant prostate cancer; EV: Extracellular vesicles; gGlu-HMRG: $\gamma$-glutamyl hydroxymethyl rhodamine green; GGT1: Gamma-glutamyltransferase 1; GSH: Glutathione; MDR1: Multi-drug resistance protein 1; NAFLD: Non-alcoholic fatty liver disease; PC: Prostate cancer; PCA3: Prostate cancer antigen 3; P-gp: P-glycoprotein; PSA: Prostate-specific antigen; PSMA: Prostate-specific membrane antigen; PVDF: Polyvinylidene difluoride; YGluAMC: gamma-glutamyl-7amido-4-methylcoumarin

\section{Acknowledgements}

We thank Mr. Tsuyoshi Maruyama at Tokyo Metropolitan Geriatric Hospital and Mr. Yasuo Hasegawa at Tokyo Metropolitan Institute of Gerontology for their technical assistance. We also thank Drs. Hiroki Tsumoto and Yuri Miura at Tokyo Metropolitan Institute of Gerontology for their assistance in proteomic analysis.

\section{Funding}

This work was supported in part by the Grant-in-Aid from The Ministry of Education, Culture, Sports, Science, and Technology of Japan (B-16H05232 to MI). The funding body did not participate in the design of the study and collection, analysis, and interpretation of data and in writing the manuscript.

\section{Availability of data and materials}

The datasets supporting the conclusions of this article are included within the article. Any request of data and material may be sent to the corresponding author.

\section{Authors' contributions}

$\mathrm{Ml}$ conceived of and directed the project and wrote the manuscript. KKaw performed experiments, analyzed data and wrote the manuscript. YF contributed to analysis and interpretation of data. YM and TA performed immunohistochemical analysis. KMi directed sample collection and analyzed data and KH, KKam, TK KMa, YK, MT and TD contributed to sample collection and interpretation of data. All authors reviewed the manuscript and approved the final version.

\section{Competing interests}

The authors declare that they have no competing interests.

\section{Consent for publication}

Not applicable.

Ethics approval and consent to participate

This study was approved by the Bioethics Committees of Gifu University and Tokyo Metropolitan Institute of Gerontology and a written informed consent was obtained from all patients.

\section{Publisher's Note}

Springer Nature remains neutral with regard to jurisdictional claims in published maps and institutional affiliations.

\section{Author details}

${ }^{1}$ Research Team for Mechanism of Aging, Tokyo Metropolitan Institute of Gerontology, 35-2 Sakae-cho, Itabashi-ku, Tokyo 173-0015, Japan.

${ }^{2}$ Department of Pathology, Tokyo Metropolitan Geriatric Hospital, 35-2

Sakae-cho, Itabashi-ku, Tokyo 173-0015, Japan. ${ }^{3}$ Department of Urology, Gifu 
University Graduate School of Medicine, 1-1 Yanagido, Gifu, Gifu 501-1193, Japan. ${ }^{4}$ Department of Urology, Tokyo Metropolitan Geriatric Hospital, 35-2 Sakae-cho, Itabashi-ku, Tokyo 173-0015, Japan. ${ }^{5}$ Department of Clinical Laboratory, Tokyo Metropolitan Geriatric Hospital, 35-2 Sakae-cho, Itabashi-ku, Tokyo 173-0015, Japan.

Received: 22 November 2016 Accepted: 25 April 2017

Published online: 05 May 2017

\section{References}

1. Colombo M, Raposo G, Thery C. Biogenesis, secretion, and intercellular interactions of exosomes and other extracellular vesicles. Annu Rev Cell Dev Biol. 2014;30:255-89.

2. Yoshioka Y, Kosaka N, Konishi Y, Ohta H, Okamoto H, Sonoda H, Nonaka R, Yamamoto $\mathrm{H}$, Ishii $\mathrm{H}$, Mori $\mathrm{M}$, et al. Ultra-sensitive liquid biopsy of circulating extracellular vesicles using ExoScreen. Nat Commun. 2014;5:3591.

3. Melo SA, Luecke LB, Kahlert C, Fernandez AF, Gammon ST, Kaye J, LeBleu VS, Mittendorf EA, Weitz J, Rahbari N, et al. Glypican-1 identifies cancer exosomes and detects early pancreatic cancer. Nature. 2015;523(7559):177-82.

4. Siegel R, Ma J, Zou Z, Jemal A. Cancer statistics, 2014. CA Cancer J Clin. 2014;64(1):9-29.

5. Kirby M, Hirst C, Crawford ED. Characterising the castration-resistant prostate cancer population: a systematic review. Int J Clin Pract. 2011;65(11):1180-92.

6. Berthold DR, Pond GR, Soban F, de Wit R, Eisenberger M, Tannock IF. Docetaxel plus prednisone or mitoxantrone plus prednisone for advanced prostate cancer: updated survival in the TAX 327 study. J Clin Oncol. 2008;26(2):242-5.

7. Tannock IF, de Wit R, Berry WR, Horti J, Pluzanska A, Chi KN, Oudard S, Theodore C, James ND, Turesson I, et al. Docetaxel plus prednisone or mitoxantrone plus prednisone for advanced prostate cancer. N Engl J Med. 2004;351(15):1502-12.

8. Beltran H, Beer TM, Carducci MA, de Bono J, Gleave M, Hussain M, Kelly WK, Saad F, Sternberg C, Tagawa ST, et al. New therapies for castration-resistant prostate cancer: efficacy and safety. Eur Urol. 2011;60(2):279-90.

9. Catalona WJ, Richie JP, Ahmann FR, Hudson MA, Scardino PT, Flanigan RC, de Kernion JB, Ratliff TL, Kavoussi LR, Dalkin BL, et al. Comparison of digital rectal examination and serum prostate specific antigen in the early detection of prostate cancer: results of a multicenter clinical trial of 6,630 men. J Urol. 1994;151(5):1283-90.

10. Catalona WJ, Partin AW, Slawin KM, Brawer MK, Flanigan RC, Patel A, Richie JP, de Kernion JB, Walsh PC, Scardino PT, et al. Use of the percentage of free prostate-specific antigen to enhance differentiation of prostate cancer from benign prostatic disease: a prospective multicenter clinical trial. JAMA. 1998;279(19):1542-7.

11. Stephan C, Ralla B, Jung K. Prostate-specific antigen and other serum and urine markers in prostate cancer. Biochim Biophys Acta. 2014;1846(1):99-112

12. Mizutani K, Terazawa R, Kameyama K, Kato T, Horie K, Tsuchiya T, Seike K, Ehara H, Fujita Y, Kawakami K, et al. Isolation of prostate cancer-related exosomes. Anticancer Res. 2014;34(7):3419-23.

13. Liu T, Mendes DE, Berkman CE. Functional prostate-specific membrane antigen is enriched in exosomes from prostate cancer cells. Int J Oncol. 2014;44(3):918-22.

14. Kato T, Mizutani K, Kameyama K, Kawakami K, Fujita Y, Nakane K, Kanimoto $Y$, Ehara $H$, Ito $H$, Seishima $M$, et al. Serum exosomal P-glycoprotein is a potential marker to diagnose docetaxel resistance and select a taxoid for patients with prostate cancer. Urol Oncol. 2015;33(9):385 e15-20.

15. Kharaziha P, Chioureas D, Rutishauser D, Baltatzis G, Lennartsson L, Fonseca P, Azimi A, Hultenby K, Zubarev R, Ullen A, et al. Molecular profiling of prostate cancer derived exosomes may reveal a predictive signature for response to docetaxel. Oncotarget. 2015;6(25):21740-54.

16. Kawakami K, Fujita Y, Kato T, Mizutani K, Kameyama K, Tsumoto H, Miura Y, Deguchi T, Ito M. Integrin beta4 and vinculin contained in exosomes are potential markers for progression of prostate cancer associated with taxaneresistance. Int J Oncol. 2015;47(1):384-90.

17. Wu HC, Hsieh JT, Gleave ME, Brown NM, Pathak S, Chung LW. Derivation of androgen-independent human LNCaP prostatic cancer cell sublines: role of bone stromal cells. Int J Cancer. 1994;57(3):406-12.

18. Thalmann GN, Anezinis PE, Chang SM, Zhau HE, Kim EE, Hopwood VL, Pathak S, von Eschenbach AC, Chung LW. Androgen-independent cancer progression and bone metastasis in the LNCaP model of human prostate cancer. Cancer Res. 1994;54(10):2577-81.
19. Thery C, Amigorena S, Raposo G, Clayton A. Isolation and characterization of exosomes from cell culture supernatants and biological fluids. Curr Protoc Cell Biol. 2006; Chapter 3:Unit 322.

20. Urano Y, Sakabe M, Kosaka N, Ogawa M, Mitsunaga M, Asanuma D, Kamiya M, Young MR, Nagano T, Choyke PL, et al. Rapid cancer detection by topically spraying a gamma-glutamyltranspeptidase-activated fluorescent probe. Sci Transl Med. 2011;3(110):110ra9.

21. Zhang H, Forman HJ, Choi J. Gamma-glutamyl transpeptidase in glutathione biosynthesis. Methods Enzymol. 2005:401:468-83.

22. Corti A, Franzini M, Paolicchi A, Pompella A. Gamma-glutamyltransferase of cancer cells at the crossroads of tumor progression, drug resistance and drug targeting. Anticancer Res. 2010;30(4):1169-81.

23. Kinlough CL, Poland PA, Bruns JB, Hughey RP. Gamma-glutamyltranspeptidase: disulfide bridges, propeptide cleavage, and activation in the endoplasmic reticulum. Methods Enzymol. 2005:401:426-49.

24. Franzini M, Bramanti E, Ottaviano V, Ghiri E, Scatena F, Barsacchi R, Pompella A, Donato L, Emdin M, Paolicchi A. A high performance gel filtration chromatography method for gamma-glutamyltransferase fraction analysis. Anal Biochem. 2008:374(1):1-6.

25. Fornaciari I, Fierabracci V, Corti A, Aziz Elawadi H, Lorenzini E, Emdin M, Paolicchi A, Franzini M. Gamma-glutamyltransferase fractions in human plasma and bile: characteristic and biogenesis. PLoS One. 2014;9(2):e88532.

26. Anderson ME. Glutathione: an overview of biosynthesis and modulation. Chem Biol Interact. 1998;111-112:1-14.

27. Hanigan $\mathrm{MH}$, Frierson HF Jr. Immunohistochemical detection of gammaglutamyl transpeptidase in normal human tissue. J Histochem Cytochem. 1996;44(10):1101-8.

28. Heisterkamp N, Groffen J, Warburton D, Sneddon TP. The human gammaglutamyltransferase gene family. Hum Genet. 2008;123(4):321-32.

29. Carter BZ, Shi ZZ, Barrios R. Lieberman MW: gamma-glutamyl leukotrienase, a gamma-glutamyl transpeptidase gene family member, is expressed primarily in spleen. J Biol Chem. 1998:273(43):28277-85.

30. Hanigan MH. Gamma-glutamyl transpeptidase: redox regulation and drug resistance. Adv Cancer Res. 2014:122:103-41.

31. Stark AA, Zeiger E, Pagano DA. Glutathione metabolism by gammaglutamyltranspeptidase leads to lipid peroxidation: characterization of the system and relevance to hepatocarcinogenesis. Carcinogenesis. 1993;14(2):183-9.

32. Pompella A, Corti A, Paolicchi A, Giommarelli C, Zunino F. Gammaglutamyltransferase, redox regulation and cancer drug resistance. Curr Opin Pharmacol. 2007;7(4):360-6.

33. Whitfield JB, Pounder RE, Neale G, Moss DW. Serum -glytamyl transpeptidase activity in liver disease. Gut. 1972;13(9):702-8.

34. Strasak AM, Pfeiffer RM, Klenk J, Hilbe W, Oberaigner W, Gregory M, Concin $H$, Diem G, Pfeiffer KP, Ruttmann E, et al. Prospective study of the association of gamma-glutamyltransferase with cancer incidence in women. Int J Cancer. 2008:123(8):1902-6.

35. Strasak AM, Rapp K, Brant $\sqcup$, Hilbe W, Gregory M, Oberaigner W, Ruttmann E, Concin H, Diem G, Pfeiffer KP, et al. Association of gammaglutamyltransferase and risk of cancer incidence in men: a prospective study. Cancer Res. 2008:68(10):3970-7.

36. Lee DS, Evans JC, Robins SJ, Wilson PW, Albano I, Fox CS, Wang TJ, Benjamin EJ, D'Agostino RB, Vasan RS. Gamma glutamyl transferase and metabolic syndrome, cardiovascular disease, and mortality risk: the Framingham heart study. Arterioscler Thromb Vasc Biol. 2007;27(1):127-33.

37. Morsi Ml, Hussein AE, Mostafa M, El-Abd E, El-Moneim NA. Evaluation of tumour necrosis factor-alpha, soluble P-selectin, gamma-glutamyl transferase, glutathione S-transferase-pi and alpha-fetoprotein in patients with hepatocellular carcinoma before and during chemotherapy. $\mathrm{Br} J$ Biomed Sci. 2006;63(2):74-8.

38. Simic T, Dragicevic D, Savic-Radojevic A, Cimbaljevic S, Tulic C, Mimic-Oka J. Serum gamma glutamyl-transferase is a sensitive but unspecific marker of metastatic renal cell carcinoma. Int J Urol. 2007;14(4):289-93.

39. Franzini M, Fornaciari I, Fierabracci V, Elawadi HA, Bolognesi V, Maltinti S, Ricchiuti A, De Bortoli N, Marchi S, Pompella A, et al. Accuracy of b-GGT fraction for the diagnosis of non-alcoholic fatty liver disease. Liver Int. 2012; 32(4):629-34.

40. Mizushima T, Ohnishi S, Shimizu Y, Hatanaka Y, Hatanaka KC, Hosono H, Kubota Y, Natsuizaka M, Kamiya M, Ono S, et al. Fluorescent imaging of superficial head and neck squamous cell carcinoma using a gamma-glutamyltranspeptidaseactivated targeting agent: a pilot study. BMC Cancer. 2016;16:411. 
41. Ueo H, Shinden Y, Tobo T, Gamachi A, Udo M, Komatsu H, Nambara S, Saito $T$, Ueda $M$, Hirata $H$, et al. Rapid intraoperative visualization of breast lesions with gamma-glutamyl hydroxymethyl rhodamine green. Sci Rep. 2015;5:12080

42. Hino H, Kamiya M, Kitano K, Mizuno K, Tanaka S, Nishiyama N, Kataoka K, Urano Y, Nakajima J. Rapid cancer fluorescence imaging using a gammaGlutamyltranspeptidase-specific probe for primary lung cancer. Transl Oncol. 2016;9(3):203-10.

43. Sato C, Abe S, Saito Y, So Tsuruki E, Takamaru H, Makazu M, Sato Y, Sasaki H, Tanaka H, Ikezawa N, et al. A pilot study of fluorescent imaging of colorectal tumors using a gamma-glutamyl-transpeptidase-activatable fluorescent probe. Digestion. 2015;91(1):70-6.

44. Frierson HF Jr, Theodorescu D, Mills SE. Hanigan MH: gamma-Glutamyl transpeptidase in normal and neoplastic prostate glands. Mod Pathol. 1997:10(1):1-6.

45. Saini S. PSA and beyond: alternative prostate cancer biomarkers. Cell Oncol (Dordr). 2016;39(2):97-106.

46. Cui Y, Cao W, Li Q, Shen H, Liu C, Deng J, Xu J, Shao Q. Evaluation of prostate cancer antigen 3 for detecting prostate cancer: a systematic review and meta-analysis. Sci Rep. 2016;6:25776.

47. Tomlins SA, Rhodes DR, Perner S, Dhanasekaran SM, Mehra R, Sun XW Varambally S, Cao X, Tchinda J, Kuefer R, et al. Recurrent fusion of TMPRSS2 and ETS transcription factor genes in prostate cancer. Science. 2005; 310(5748):644-8.

48. Leyten GH, Hessels D, Jannink SA, Smit FP, de Jong H, Cornel EB, de Reijke TM, Vergunst $\mathrm{H}$, Kil P, Knipscheer BC, et al. Prospective multicentre evaluation of PCA3 and TMPRSS2-ERG gene fusions as diagnostic and prognostic urinary biomarkers for prostate cancer. Eur Urol. 2014;65(3):534-42.

49. Huang X, Yuan T, Liang M, Du M, Xia S, Dittmar R, Wang D, See W, Costello BA, Quevedo F, et al. Exosomal miR-1290 and miR-375 as prognostic markers in castration-resistant prostate cancer. Eur Urol. 2015;67(1):33-41.

50. Van Hemelrijck M, Jassem W, Walldius G, Fentiman IS, Hammar N, Lambe M, Garmo H, Jungner I, Holmberg L. Gamma-glutamyltransferase and risk of cancer in a cohort of 545,460 persons - the Swedish AMORIS study. Eur J Cancer. 2011:47(13):2033-41.

\section{Submit your next manuscript to BioMed Central} and we will help you at every step:

- We accept pre-submission inquiries

- Our selector tool helps you to find the most relevant journal

- We provide round the clock customer support

- Convenient online submission

- Thorough peer review

- Inclusion in PubMed and all major indexing services

- Maximum visibility for your research

Submit your manuscript at www.biomedcentral.com/submit 